\title{
Hierarchical Organizations and a Supporting Software Architecture for Floating Car Data
}

\author{
Robrecht Haesevoets, Danny Weyns, Tom Holvoet, Wouter Joosen \\ Department of Computer Science \\ Katholieke Universtiteit Leuven \\ Leuven, Belgium \\ Email: Robrecht.Haesevoets@cs.kuleuven.be
}

\author{
Paul Valckenaers \\ Department of Mechanical Engineering \\ Katholieke Universtiteit Leuven \\ Leuven, Belgium \\ Email:Paul.Valckenaers@mech.kuleuven.be
}

\begin{abstract}
The use of floating car data is an interesting method to monitor traffic. Vehicles act as local traffic sensors and data from individual vehicles is aggregated into higher-level information. We propose a number of reusable organization abstractions and a software architecture to support a multi-agent approach applied to floating car data. The abstractions are based on the idea of hierarchical organizations which are used as units of data aggregation. In this approach, an agent is deployed on each vehicle. At the lowest level, nearby vehicle agents collaborate to aggregate individual traffic data and distribute it to local clients such as traffic light controllers. At higher-levels, organizations are built up from lower-level organizations and represent specific aggregation interests such as the total congestion level in a specific area. A prototype was built, supporting a two-level organization structure, and is used in a simulated traffic environment as initial validation.
\end{abstract}

\section{Introduction}

Recent technological advances, such as onboard car computers and inter-vehicle communication, allow exploring new approaches for traffic monitoring. A promising approach is the use of floating car data in which vehicles are used as local traffic sensors and individual traffic data is aggregated [10]. This paper considers a multi-agent approach applied to floating car data. An agent is deployed on each vehicle, which has access to sensors installed on the vehicle, allowing the agent to get local context information such as GPS location, speed and driving direction. A wireless communication unit on each vehicle allows for local interactions. Nearby vehicle agents collaborate to aggregate individual traffic data and distribute it to interested clients, such as traffic light controllers or driver assistance systems. This decentralized approach avoids the bottleneck of a centralized control center and makes the system more robust and scalable.
However, the large amount of interacting vehicle agents and the dynamic nature of the problem, in which vehicles constantly come and go, lead to complex interaction and coordination problems. In order to deal with these complexities we offer a number of organization abstractions and a supporting software architecture.

The organization abstractions presented in this paper build upon earlier work [6]. Agents are grouped in organizations in which they play roles. A coordination infrastructure is responsible for managing the organization dynamics, resulting in the separation of two concerns: (1) the management of organization dynamics and (2) the actual functionality provided by the agents playing roles. This separation reduces the complexity of the agents, making it easier to understand, design, and manage organizations in multi-agent systems. In contrast with the work in [6], this paper uses a dynamic mobile platform (moving vehicles) instead of static road side cameras. This is reflected in a more lightweight software architecture, and an adapted organization model with support for hierarchical organizations. Hierarchical organizations are used as units of data aggregation to increase scalability.

A prototype based on the software architecture was built and is used in a simulated environment to evaluate the proposed organization abstractions.

Overview of the paper. The next section introduces the organization abstractions. Section 3 presents a software architecture supporting these abstractions. Section 4 describes how the software architecture uses the abstractions to handle organization dynamics. We describe the prototype and evaluation in Sect. 6. Section 7 discusses related work. Finally, we draw conclusions and outline issues for future research.

\section{Organization Abstractions}

Hierarchical organizations are used as units of data aggregation. At the lowest level, organizations consist of small groups of agents, such as nearby vehicle agents in the same traffic situation. At higher-levels, organizations are 


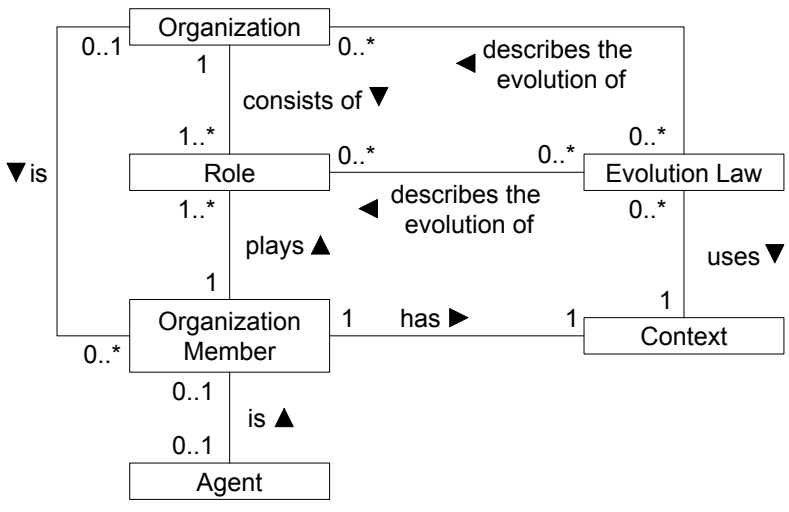

Figure 1. Overview of the organization model.

built up from lower-level organizations, representing specific aggregation interests, such as all vehicles in a traffic jam or the total congestion level on an important highway. Within organizations, agents play roles to fulfill the requirements of the organization, for example, monitoring the traffic or aggregating data. An overview of the organization abstractions is given in Fig. 1.

\subsection{Organizations}

Organizations consist of organization members. Both agents and organizations can be organization members, allowing hierarchical organizations. Evolution laws describe the organization dynamics using the current context as input (see below). The concept of organization offers a higherlevel abstraction to application developers, can be used to limit information flooding, and increases the granularity of data and the scalability of data aggregation.

\subsection{Roles}

Roles are played by organization members (agents and organizations) and represent a coherent set of functionalities. The roles within an organization are defined by the evolutions laws. A number of default roles are needed to support the organization structure: context manager and organization mediator. The context manager role is played by each organization member and is responsible to keep its own context consistent with the context of the other organization members. There is one organization mediator role in every organization. This mediator is responsible for playing or delegating the roles the organization has to play as an organization member in higher-level organizations. Apart from default roles, application specific roles can be used within organizations, such as a data aggregation in the traffic monitoring case.

\begin{tabular}{|c|c|c|}
\hline $\begin{array}{l}\text { for each } \\
\text { member }\end{array}$ & $\begin{array}{l}\text { if member belongs } \\
\text { to an organization }\end{array}$ & $\begin{array}{c}\text { if member is mediator } \\
\text { of an organization }\end{array}$ \\
\hline $\begin{array}{l}\text { Member (mem): } \\
\text { - ID } \\
\text { - level } \\
\text { - evolutionData } \\
\text { - nbOfAgents } \\
\text { - playedRoles }\end{array}$ & $\begin{array}{l}\text { Organization (org): } \\
\text { - ID } \\
\text { - level } \\
\text { - evolutionData } \\
\text { - nbOfAgents } \\
\text { - mediatorID } \\
\text { - playedRoles }\end{array}$ & $\begin{array}{l}\text { Evolution Candidates (candidate[]): } \\
\text { - ID } \\
\text { - level } \\
\text { - evolutionData } \\
\text { - nbOfAgents } \\
\text { - mediatorID }\end{array}$ \\
\hline
\end{tabular}

Figure 2. The context of an organization member.

\subsection{Context}

Each organization member has an associated context representing the current state of its local environment and organization. An overview of the context of an organization member is shown in Fig. 2. This context contains data on the member itself and the organization it belongs to. If the member is mediator of its organization, it also contains a list of evolution candidates. Evolution candidates are other organizations this organization can cluster with, for example, by merging or joining (see Sect. 2.4).

For decentralized traffic monitoring, the evolution data consists of the current velocity, traffic state and location. At agent or vehicle level, the velocity is the vehicles velocity, the traffic state is undefined, and the location the current gps location. At higher levels the velocity and location are calculated as the average of the individual velocities and locations of all members. The traffic state has three possible values, free flow, bound flow, and congestion. The traffic state is calculated from the average velocity and density ${ }^{1}$.

\subsection{Evolution Laws}

Evolution laws define how organizations evolve, based on the context of the organization members. There are currently five types of laws: a merge law defining how organizations of equal levels can merge, a join law defining how a lower-level organization can join a higher-level organization, a creation law defining how an organization can create a higherlevel organization, a split law defining how organization members can split from their current organization, and a role law defining the roles within an organization. These laws are chosen as a minimal set to support hierarchical organizations. Figure 3 shows an example of splitting and merging organizations. A simplified version, in pseudo code, of a merge law is given in Fig. 4.

\section{Supporting Software Architecture}

Figure 5 shows an overview of the software components deployed on each vehicle. Vehicles are connected by a

1. The density is calculated as the number of vehicles per length unit, which is derived from the individual locations of the members. A hysteresis curve can be used to avoid rapid fluctuations. 


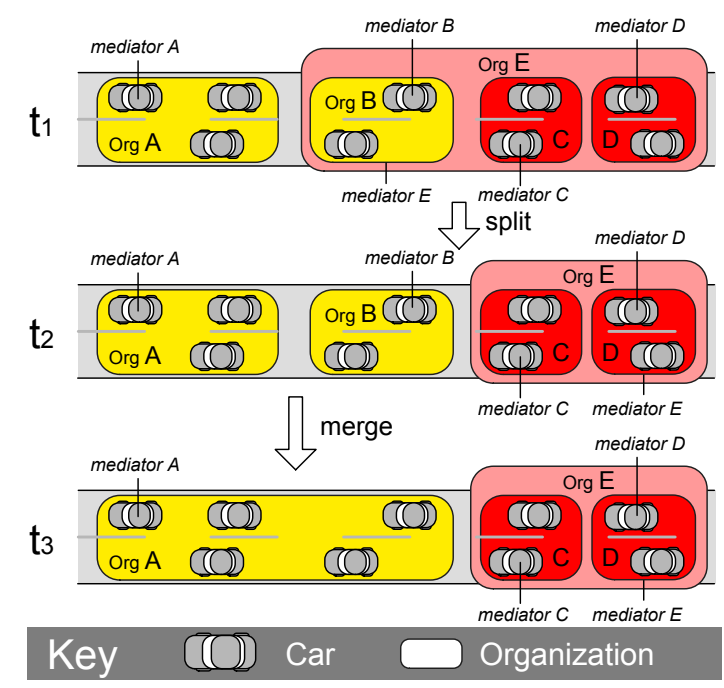

Figure 3. Examples of how organizations can evolve, as defined by the evolution laws.

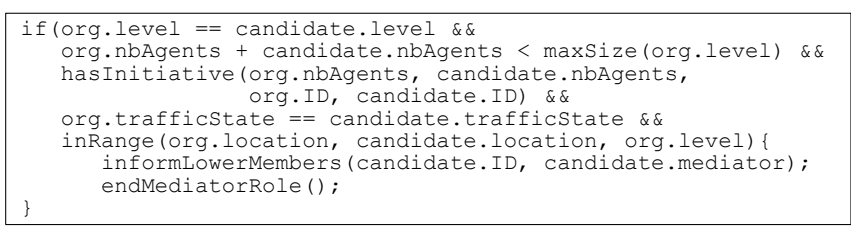

Figure 4. A merge law for the traffic monitoring case in pseudo code.

wireless communication network. The software on each vehicle consists of the Host Infrastructure, the Coordination Infrastructure and the Agent. The host infrastructure offers access to the operating system and other low level services. As in [6], the coordination infrastructure is responsible for the management of organization dynamics and offers organizations and roles to the agent. The abstractions of organization and role allow to separate the management of organization dynamics from the actual functionality provided by the agents playing roles. Agents can communicate and perceive the world through the roles they play.

The core of the coordination infrastructure consists of the organization controller. This controller uses the context in the Local Context repository as input, to evolve the organizations, based on the Organization Laws. Organizations are managed by updating the local context. Roles are managed through the role life cycle interface, which allows to add and remove roles from the Role Pool. All roles have access to the perception and communication module and can update and view the local context. Agents have to play the roles in the role pool and have access to communication and perception services through the role interfaces of these roles.

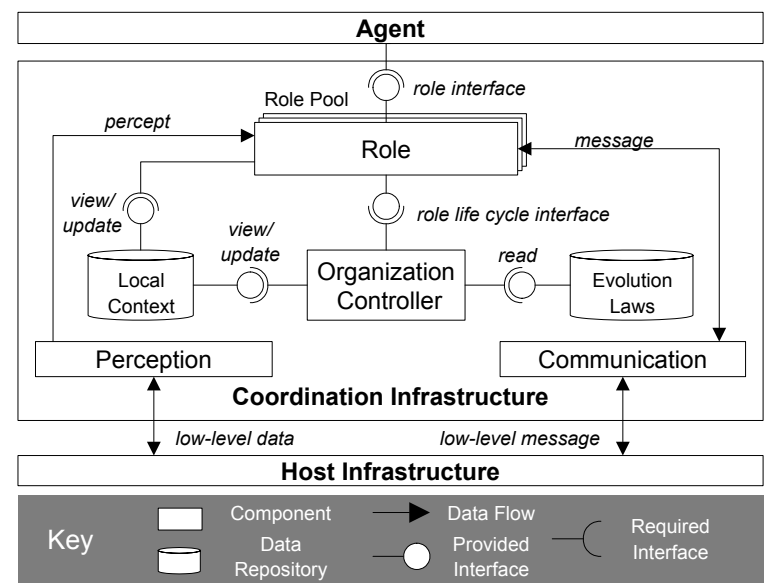

Figure 5. Components of the supporting software architecture on one vehicle.

Context Management. The context of each organization member is maintained in a local context repository. The context of an agent is maintained in the local context repository on the node it is deployed on (e.g. a vehicle). The context of an organization is maintained in the same repository as its mediator's context ${ }^{2}$. The context of an organization member is kept up to date by the context manager and the organization mediator roles. Context manager roles send out periodic context messages to each other within the organization. With these context messages, each member can calculate the aggregated evolution data. Depending on the application, specific aggregation policies have to be provided. Organization mediator roles send context messages to their members, containing data on the current id of the organization and mediator, and messages to other mediators within a certain range (e.g. hop-count), to maintain a list of potential evolution candidates.

\section{Organization Dynamics}

The organization controller on each vehicle uses the local context and the evolution laws, to evolve the organizations ${ }^{3}$. The evolution laws are executed locally, meaning, there is no direct negotiation between different controllers to decide what to $\mathrm{do}^{4}$. The execution of a law is first reflected locally in changes of the local context, and subsequently in context messages that are sent out. The eventual structure of the organization emerges from the context messages received by other organization members. The mediator of

2. The mediator of an organization can be another organization, but at the lowest level it is always an agent.

3. Currently the laws are evaluated sequentially in random order on all evolution candidates. The creation law ensures an agent is always in an organization.

4. Evolution laws are defined to avoid or correct from inconsistent states. E.g., in absence of a mediator, a new mediator is elected, as defined by the role law. 


\begin{tabular}{|c|c|c|}
\hline \multicolumn{3}{|c|}{ context mediator $B$} \\
\hline $\begin{array}{l}\text { Member: } \\
\ldots\end{array}$ & $\begin{array}{l}\text { Ogranization: } \\
- \text { ID = orgB } \\
\text { - level }=1 \\
\text { - trafficState }=\text { bound flow } \\
\text { - location }=\left(x \_B, y \_B\right) \\
\text { - nbOfAgents }=2 \\
\text { - mediator = mediatorB } \\
\ldots\end{array}$ & $\begin{array}{l}\text { Evolution Candidates: } \\
- \text { ID }=\text { orgA } \\
\text { - level }=1 \\
\text { - trafficState }=\text { bound flow } \\
\text { - location }=\left(x \_A, y \_A\right) \\
\text { - nbOfAgents }=3 \\
\text { - mediator }=\text { mediatorA } \\
\ldots\end{array}$ \\
\hline
\end{tabular}

Figure 6. Extract of the context of mediator $\mathrm{B}$ at $t_{2}$.

an organization is responsible for reporting the changes to its lower-level organization members. We now explain the merge of organizations $\mathrm{A}$ and $\mathrm{B}$, as shown in Fig. 3, in detail.

Figure 6 shows an extract of the context of mediator $\mathrm{B}$ at $t_{2}$, in which organization $\mathrm{A}$ is an evolution candidate for organization B. The organization controller of mediator B evaluates the merge law with organization $\mathrm{A}$ as candidate. According to the context, organization A is the same level as organization $\mathrm{B}$, has the same traffic state and is within range of organization B. The merge law can thus be executed if the initiative condition is satisfied. Because organization B has less agents than organization $\mathrm{A}$, the initiative condition is only satisfied for organization B. The organization controller of mediator $\mathrm{B}$ initiates the merge while a concurrent merge of the controller of mediator $\mathrm{A}$ is avoided. To execute the merge, the context of mediator B is adapted by the controller, stating a new organization id, organization $\mathrm{A}$, and a new mediator, mediator A. This context change is propagated to the lower organization members, through context messages sent out by mediator B. The lower organization members update their context and start sending context messages to mediator A and the other members of organization A. Soon, the new organization structure emerges in the context of all members of organization $\mathrm{A}$, as shown at $t_{3}$.

\section{Data Aggregation}

Organizations can be used as units of data aggregation, in which agents can perform specific aggregation tasks, increasing the granularity of data and the scalability. An example is shown in Fig. 7. At the lowest-level (level 1 ), organizations consist of nearby vehicles and individual traffic data is aggregated into traffic data for the whole group. At higher-levels, organizations can be used to further increase the granularity of the data (level 2), or they can represent specific aggregation interests, such as all traffic data for a certain region (level 3). At each level of the organization, there can be a number of distribution roles, responsible for distributing the aggregated traffic data to interested clients, such as traffic light controllers, driver assistance systems or emergency services.

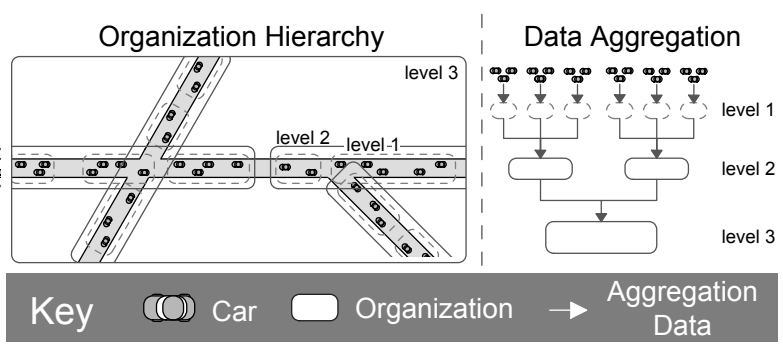

Figure 7. An example of an hierarchical organization, used for data aggregation.

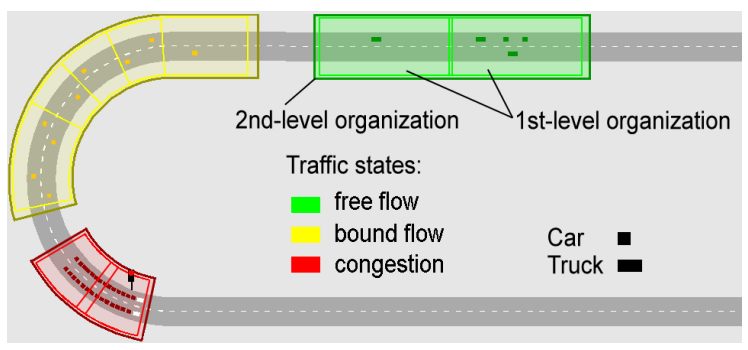

Figure 8. A screenshot of the prototype.

\section{Prototype and Evaluation}

As initial validation, a prototype based on the software architecture was built, supporting a two-level organization structure. The evaluation focuses on the validation of the hierarchical organization concepts and its bandwidth usage. The host infrastructure and wireless network are replaced by a communication network and traffic simulator ${ }^{5}$. The evaluations use a simulated two-lane highway of 1000 meters, shown in Fig. 8. Communication between vehicles is limited to 150 meters. Each data point in the experiments, represents the average of 600 individual measurements, together with a 95 percentage confidence interval.

In a first experiment, the range, defining the maximum size, of the first-level organizations is 150 meters, while the number of vehicles on the highway varies. Figure 9 (top) shows the average amount of messages to be processed per vehicle agent as a function of the number of vehicles. The total number of messages and messages related to different parts of the context are shown. A linear relationship can be observed between the amount of messages to be processed and the number of vehicles on the highway.

In a second experiment, the total number of vehicles is kept at 80 , while changing the range of the first-level organizations. Figure 9 (bottom) shows the average amount of messages to be processed per vehicle agent in function of the range of first-level organizations. As the range of the first-level organizations increases, the number of vehicle

5. Our traffic simulator is based on an existing traffic simulator by $\mathrm{M}$. Treiber, http://www.traffic-simulation.de 

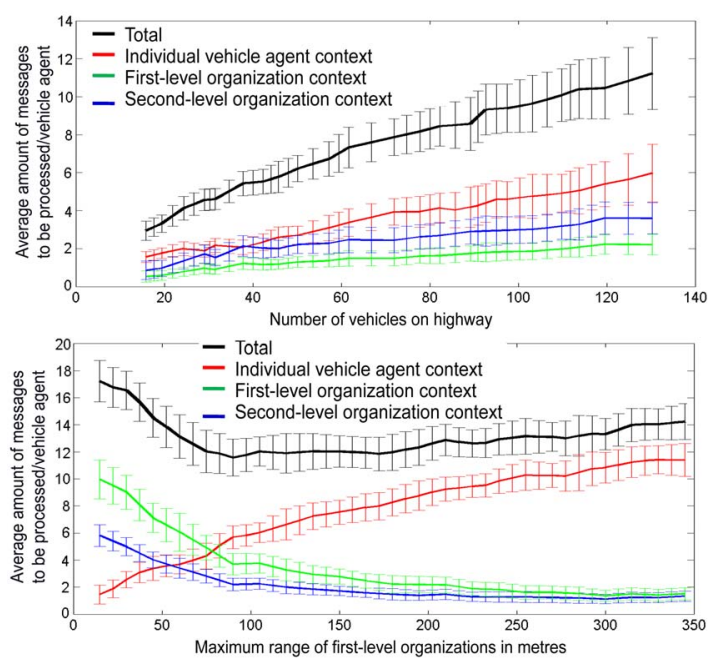

Figure 9. Average amount of messages processed per vehicle agent in function of: the number of vehicles on the highway (top), the range of first-level organizations (bottom).

agents per first-level organization increases, reflected in an increase in the number of messages related to vehicle agent context. The amount of messages related to firstlevel organizations decreases, because as their size increases. This results in a decrease of first-level organizations in second-level organizations, which is reflected in a decrease of messages related to second-level organizations.

The test results show that different levels of hierarchical organizations are created, and indicate that the system scales well with respect to bandwidth usage for an increasing number of vehicles on the highway.

\section{Related Work}

This section first discusses related work on inter-vehicle and mobile communication and its use in traffic applications. We then contrast our work with the more traditional traffic monitoring approaches. Next, we discuss organizations in Multi-Agent systems, and finally we discuss the use of organizational concepts in mobile settings.

Inter-Vehicle and Mobile Communication. Inter-vehicle communication and car networks are an important component of intelligent transportation systems and an active research domain [12]. Car networks are typically conceived as vehicular ad hoc networks [14]. Peer-to-peer networks have been used in car networks for traffic safety application such as collision avoidance [3]. In contrast to our work, most of the approaches focus on local traffic situations within a limited area, for example nearby cars at intersections or areas around an obstacle. By using hierarchical concepts, our approach tries to enhance the scalability of the data aggregation and distribution.

In the area of wireless sensor networks, routing protocols for a mobile setting [2] and hierarchical protocols [7] have been proposed. The clustering methods in these hierarchical protocols, however, are based on specific sensor related data such as the battery power and signal strength. The focus of our work, is not on specific communication or routing protocols but on using a mobile communication infrastructures to support higher-level coordination structures, such as hierarchical organizations.

Traditional Traffic Monitoring. Traffic monitoring is an extensively studied field of research. Traditional approaches typically consist of vision-based traffic surveillance [9]. There is an increasing interest in the traffic monitoring research community for multi-sensor fusion [1]. Data from possibly unsynchronized sensors and delayed observations is aggregated into data of higher quality and reliability. In contrast to approaches based on inter-vehicle communication, these approaches assume a static road infrastructure, which is often more expensive to maintain and will more likely lead to a central bottleneck.

Organizations in Multi-Agent Systems. Roles and organizations are generally acknowledged as valuable abstractions to build multi-agent systems [8]. A particular line of related research are computational institutions [5]. Using laws and norms, a middleware structure defines the currently permitted actions of agents. A number of approaches exist to support organizational evolution. AGRE [4] (Agent Group Role Environment), offer primitives for agents to join groups and play roles. TuCSoN [13] offers programmable tuplespaces. Organizational settings can be changed by agents, by reading and modifying the tuple contents. In contrast to our approach, agents are responsible for managing the organization structure. Both approaches offer no explicit support to model evolutional changes at the inter-organization level.

In our work, we show how a coordination infrastructure enables to separate the management of organization dynamics for the functionality associated with the roles played in the organization, spreading the complexity over the coordination infrastructure and the agents.

Organizations in Mobile Applications. [3] describes a communication system for traffic safety systems. Nearby vehicles are grouped in peer spaces, representing vehicles interested in the same local safety issues. [11] defines proximity groups. Proximity groups take both location and functional aspects into account. Entities join proximity groups based on their location and their functional interests. In contrast to our work, approaches supporting organization concepts in mobile applications typically focus on local organizations and short ranged safety information. We offer support for 
both local and larger organizations by using the notion of hierarchical organizations.

\section{Conclusions and Future Work}

In this paper we presented a number of organization abstractions and a supporting software architecture, based on the idea of hierarchical organizations, which can be used as units of data aggregation. At different organization levels, the individual data of organization members can be aggregated, hiding the details of individual data and improving scalability. The presented coordination infrastructure offers higher-level abstractions to application developers.

The coordination infrastructure is responsible for the evolution of the organizations. The abstractions of organization and role separate the evolution of dynamic organizations from the actual functionality provided by the agents playing roles. Separating these two concerns reduces the complexity of the agents, making it easier to understand, design, and manage organizations in multi-agent systems. In most of the existing approaches, supporting organizations in multi-agent systems, agents themselves are responsible for managing the organizations and roles. Approaches supporting organization concepts in mobile applications, typically focus on local organizations. We offer support for both local and larger organizations by using the notion of hierarchical organizations.

We presented a prototype, supporting a two-level organization structure, and we used it in a simulated traffic environment for evaluation. Experiments show the creation of hierarchical organizations and indicate that the approach scales well with respect to bandwidth usage. The amount of messages to be processed by individual agents can be minimized by choosing an optimal size for the low-level organizations.

Future Work. Communication gaps between vehicles, and relaxing the assumption that a vehicle agent is deployed on every vehicle have not yet been studied in detail. Our current prototype only supports two levels of organizations. This could be increased and optimized, further enhancing the scalability of our approach. Formalization of the organization model and evolution laws can be interesting to formally proof the clustering behavior of agents and organizations.

\section{Acknowledgement}

This research is partially funded by the Interuniversity Attraction Poles Programme Belgian State, Belgian Science Policy, and by the Research Fund K.U.Leuven. Danny Weyns is supported by the Foundation for Scientific Research in Flanders (FWO-Vlaanderen).

\section{References}

[1] Iyad Abuhadrous, Fawzi Nashashibi, and Claude Laurgeau. Multi-Sensor Fusion (GPS, IMU, Odometers) for Precise Land Vehicle Localisation Using RTMAPS. In 11th International Conference on Advanced Robotics (ICAR 2003), 2003.

[2] K. Akkaya and M. Younis. A survey on routing protocols for wireless sensor networks. Ad Hoc Networks, 3(3):325-349, 2005.

[3] L. Chisalita and N. Shahmehri. A peer-to-peer approach to vehicular communication for the support of traffic safety applications. Intelligent Transportation Systems, 2002. Proceedings. The IEEE 5th International Conference on, pages 336-341, 2002.

[4] J. Ferber, F. Michel, and J. Baez. AGRE: Integrating environments with organizations. In First International Workshop on Environments for Multi-Agent Systems, volume 3374 of Lecture Notes in Computer Science, New York, NY, USA, 2005. Springer-Verlag.

[5] A. Garca-Camino, P. Noriega, and J .Rodrguez-Aguilar. Implementing norms in electronic institutions. In AAMAS '05: Proceedings of the fourth international joint conference on Autonomous agents and multiagent systems, New York, NY, USA, 2005. ACM Press.

[6] R. Haesevoets, B. Van Eylen, D. Weyns, A. Helleboogh, T. Holvoet, and W. Joosen. Managing Agent Interactions with Context-Driven Dynamic Organizations. In Engineering Environment-Mediated Multiagent Systems, Lecture Notes in Computer Science. Springer-Verlag, 2007.

[7] WR Heinzelman, A. Chandrakasan, H. Balakrishnan, and C. MIT. Energy-efficient communication protocol for wireless microsensor networks. System Sciences, 2000.

[8] N. Jennings. On agent-based software engineering. Artificial Intelligence, 177(2):277-296, 2000.

[9] Y.K. Jung, K.W. Lee, and Y.S. Ho. Content-based event retrieval using semantic scene interpretation for automated traffic surveillance. ITS, 2(3):151-163, September 2001.

[10] BS Kerner, C. Demir, RG Herrtwich, SL Klenov, H. Rehborn, M. Aleksi, and A. Haug. Traffic state detection with floating car data in road networks. In Intelligent Transportation Systems, 2005. Proceedings. 2005 IEEE, pages 700-705, 2005.

[11] M. Killijian, R. Cunningham, R. Meier, L. Mazare, and V. Cahill. Towards group communication for mobile participants. Proceedings of the 1st ACM Workshop on Principles of Mobile Computing (POMC 2001), pages 75-82, 2001.

[12] J. Luo and J.P. Hubaux. A Survey of Inter-Vehicle Communication. EPFL, Lausanne, Switzerland, Tech. Rep, 2004.

[13] A. Omicini and A. Ricci. Reasoning about organisation: Shaping the infrastructure. AI* IA Notizie, 16(2):7-16, 2003.

[14] S. Yousefi, MS Mousavi, and M. Fathy. Vehicular Ad Hoc Networks (VANETs): Challenges and Perspectives. ITS Telecommunications Proceedings, 2006 6th International Conference on, pages 761-766, 2006. 